\title{
Parámetros genéticos de caracteres asociados a la uniformidad del diámetro de fibra en alpacas Huacaya en Puno, Perú
}

\author{
Genetic parameters of characters associated with the uniformity of fibre diameter \\ in alpacas Huacaya in Puno, Peru
}

\author{
Herbert Aguilar ${ }^{1,2,4}$, Gustavo Gutiérrez R., ${ }^{1,2}$, María Wurzinger ${ }^{3}$
}

\section{Resumien}

\begin{abstract}
El objetivo del presente estudio fue estimar los parámetros genéticos (heredabilidad y correlaciones genéticas) para caracteres asociados a la uniformidad del diámetro de fibra en el vellón de alpacas tuis del fundo Mallkini (Puno, Perú). Se utilizaron muestras de las zonas corporales de muslo, costillar medio y paleta de 1127 animales a la primera esquila ( 573 machos y 554 hembras) nacidos en 2015 y 2016. Las muestras fueron analizadas bajo la norma IWTO-12 del equipo Sirolan Laserscan. El archivo de pedigrí contaba con 10481 alpacas dando un coeficiente de consanguinidad de $0.16 \%$. Los parámetros genéticos se estimaron mediante los softwares ASReml y Pedigree Viewer. Los caracteres evaluados fueron el diámetro de fibra (DF) y su desviación estándar (DE), coeficiente de variación $(\mathrm{CV})$ y factor de confort $(\mathrm{FC})$. El análisis genético se realizó utilizando un modelo animal multivariado, que incluye el efecto genético aditivo del animal como efecto aleatorio; año de esquila, punta y sexo como efectos fijos, y edad de esquila como covariable lineal. Las heredabilidades para todas las características por zona fueron moderadas $(0.12$ a 0.38$)$ y para las zonas en conjunto resultaron también moderadas, excepto para el coeficiente de variación $(0.17$ a 0.43$)$. La correlación genética entre el diámetro de fibra y la desviación estándar mostró un valor elevado y positivo (0.80), mientras que para el factor de confort fue alto y negativo (-0.93) y para el coeficiente de variación fue negativo y bajo (-0.06). En conclusión, existe un alto grado de correlación genética entre el diámetro de fibra (DF) y su desviación estándar (DE), de modo que seleccionando por ambos caracteres se lograría reducir la variabilidad de finura en los vellones.
\end{abstract}

Palabras clave: alpaca; parámetros genéticos; diámetro de fibra; ASReml

\footnotetext{
${ }^{1}$ Escuela de Posgrado, Universidad Nacional Agraria La Molina, Lima, Perú

${ }^{2}$ Facultad de Zootecnia, Departamento de Producción Animal, Universidad Nacional Agraria La Molina, Lima, Perú

${ }^{3}$ University of Natural Resources and Life Sciences, Vienna (BOKU), Austria

${ }^{4}$ E-mail: herbertstree@hotmail.com
}

Recibido: 22 de octubre de 2018

Aceptado para publicación: 10 de julio de 2019 
The aim of this study was to estimate the genetic parameters (heritability and genetic correlations) for characters associated with the uniformity of fibre diameter in fleece of young (tuis) alpacas of the Mallkini farm (Puno, Peru). Samples were taken from the thigh, mid-rib and shoulder of 1127 animals at the first shearing (573 males and 554 females) born in 2015 and 2016. The samples were analysed under the IWTO-12 standard of the Sirolan Laserscan equipment. The pedigree file had 10481 alpacas giving a coefficient of inbreeding of $0.16 \%$. The genetic parameters were estimated using ASReml and Pedigree Viewer software. The characters evaluated were the fibre diameter and its standard deviation, coefficient of variation and comfort factor. The genetic analysis was carried out using a multivariate animal model, which includes the additive genetic effect of the animal as a random effect; year of shearing, group/category and sex as fixed effects, and age of shearing as a linear covariable. The heritability for all the characteristics by body area was moderate $(0.12$ to 0.38$)$ and for the mean of the three body areas was also moderate, except for the coefficient of variation ( 0.17 to 0.43$)$. The genetic correlation between the fibre diameter and the standard deviation showed a high and positive value (0.80), while for the comfort factor it had a high and negative correlation (-0.93) and for the coefficient of variation was negative and low (-0.06). In conclusion, there is a high degree of genetic correlation between the fibre diameter and its standard deviation, so that selecting for both characters would reduce the variability of fineness in the fleeces.

Key words: alpaca; genetic parameters; fiber diameter; ASReml

\section{INTRODUCCIÓN}

El Perú ocupa el primer lugar en el mundo en la población de alpacas y vicuñas, y el segundo en llamas, después de Bolivia, así como cerca del $90 \%$ de la producción de fibra de camélidos sudamericanos (FAO, 2005). La producción de fibra es la principal actividad y el pilar de la economía de la población altoandina.

La uniformidad de la fibra de la alpaca está directamente relacionada con la media del diámetro de fibra (DF). La clasificación de los vellones se basa principalmente en esta característica, ya que permite una mejor valorización al momento de la comercialización; es decir, vellones de mejor uniformidad tienen mejores precios. Existen, además, otras características asociadas a la uniformidad de la fibra; entre ellas, la desviación estándar
(DE) y el coeficiente de variación (CV). Esta última es una medida de amplitud relativa del diámetro de la fibra alrededor de la media dentro de un vellón, de manera que un vellón con CV bajo indica una mayor uniformidad relativa de los diámetros de las fibras individuales que lo componen, produciendo un hilo más resistente (Manso, 2011). Otra característica es el factor de confort (FC) o factor de comodidad, entendiéndose que a mayor confort se tiene fibras de menor diámetro (Quispe et al., 2013). Consecuentemente, la uniformidad podría ser la cualidad más destacada del vellón y debería ser más estudiada para una mejor selección de las alpacas.

El objetivo del presente estudio fue estimar los parámetros genéticos de índice de herencia y correlaciones genéticas en las características diámetro de fibra, desviación estándar, coeficiente de variación y factor de confort de tres zonas corporales en alpacas tuis Huacaya a primera esquila. 


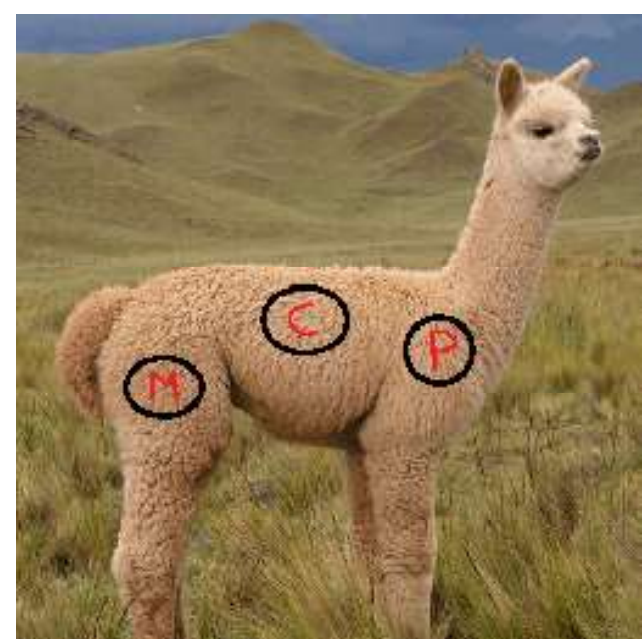

Figura 1. Puntos anatómicos de las muestras de fibra. M: muslo; C: costillar medio; p: paleta

\section{Materiales y MéTodos}

Se tomaron muestras de fibra $(20 \mathrm{~g}) \mathrm{de}$ las zonas corporales de muslo, costillar medio y paleta (Figura 1) de 1127 alpacas a la primera esquila (573 machos y 554 hembras), nacidos en los años 2015 y 2016 en el fundo Mallkini, ubicado en Puno, Perú.

Las características de la fibra en estudio asociadas a la uniformidad del vellón fueron el diámetro promedio de fibra, la desviación estándar y el coeficiente de variación del diámetro de fibra, y el factor confort (FC). Estas características fueron medidas en cada muestra de fibra utilizando la norma IWTO12 y el equipo Sirolan Laserscan de la empresa ITEC Innovation Ltd (Reino Unido), obtenido por el Programa de Ovinos y Camélidos Sudamericanos (POCA) de la Universidad Nacional Agraria la Molina (UNALM), en Lima, Perú.

Se realizó un análisis descriptivo de los datos por zona corporal, sexo, año de esquila y punta (lote de animales), estimando el promedio, la desviación estándar y el coeficien- te de variación para las variables estudiadas. Luego se realizó un análisis de consistencia para detectar valores atípicos, seguidamente se evaluó el cumplimiento de los supuestos de normalidad, homogeneidad de varianzas e independencia de errores. Para ello se utilizó un modelo fijo considerando los efectos de año $(2015,2016)$, punta (plantel, majada I y majada II), sexo (macho, hembra) y sus respectivas interacciones. Además, se incluyó la edad a la esquila como covariable lineal.

Los efectos fijos, el efecto aleatorio (animal) y las covariables a ser incluidos en el modelo animal fueron definidos según el grado de significancia utilizando el método de mínimos cuadrados ordinarios por medio del procedimiento PROC MIXED de SAS v. 9.4. Posteriormente fue creada una matriz de parentesco considerando seis generaciones, usando el archivo de genealogía de 10481 alpacas con un coeficiente de consanguinidad promedio de $0.16 \%$.

Las covarianzas y heredabilidades fueron estimadas mediante el modelo animal multivariado, utilizando la metodología de estimación de los parámetros genéticos del tipo frecuentista basada en el Método de Máxima Verosimilitud Restringida (REML), descrito por Thompson (2008), usando el programa ASReml v. 4.1 (Gilmour et al., 1995).

La ecuación del modelo animal: $y=X \beta$ $+\mathrm{Zu}+\mathrm{e}$, realizado para la estimación REML multicarácter de las cuatro características de forma conjunta; donde $y$ es el vector de observaciones; $\beta$ es el vector de efectos fijos que incluyó: el año ( 2 niveles), punta ( 3 niveles), sexo (2 niveles), y la edad al muestreo en días como covariable lineal; u es el vector representando los efectos genéticos aditivos, e es el vector de los residuales, $\mathrm{X}, \mathrm{Z}$ son las matrices de incidencia para los efectos fijos y aleatorios. Los efectos aleatorios se consideran independientes con una distribución normal de media cero y varianzas: $\operatorname{Var}(\mathrm{u})=$ $\mathrm{A} \sigma 2 \mathrm{~A}$ y $\operatorname{Var}(\mathrm{e})=\mathrm{I} \sigma 2 \mathrm{e}$ que son respectivamente las matrices de varianzas y covarianzas aditivas y residuales, donde $\mathrm{A}$ es la matriz 
numerador de relaciones aditivas, e I la matrizidentidad.

\section{Resultados y Discusión}

\section{Heredabilidades}

Las estimaciones de las heredabilidades y correlaciones genéticas para el diámetro, desviación estándar y coeficiente de variación de la fibra y el factor de confort con el promedio de las tres zonas corporales se muestran en el Cuadro 1. La mayor heredabilidad se obtuvo para el diámetro de fibra (0.43), mientras que la correlación genética más baja y negativa fue para el diámetro de fibra - coeficiente de variación (-0.06).

\section{Diámetro de Fibra}

Valores de heredabilidad del diámetro de fibra reportados en la literatura se muestran en el Cuadro 2. En líneas generales, se puede apreciar que la heredabilidad para este carácter es alta, lo que sugiere una respuesta esperada alta a la selección si se utiliza como criterio de selección, ya que el promedio del diámetro de fibra indica la finura del animal (Gutiérrez et al., 2009).

\section{Desviación Estándar}

Valores de heredabilidad de la desviación estándar del diámetro de fibra reportados en la literatura se muestran en el Cuadro 2. La heredabilidad encontrada en el presente estudio (0.31) puede ser considerada como moderada-alta. Estos resultados sugieren que habría una buena respuesta esperada a la selección para este carácter si se utiliza como criterio de selección, ya que para seleccionar por uniformidad el vellón, la desviación estándar da un indicador de la homogeneidad o variabilidad de la muestra del animal, entendiéndose que a mayor sea, mayor será la dispersión de los valores de la distribución respecto a la media y, por tanto, la media será menos representativa de las observaciones de dicha distribución (Cruz, 2011).

\section{Coeficiente de Variación}

La heredabilidad para coeficiente de variación obtenida (0.17) fue baja, comparando con las investigaciones reportadas en alpacas Huacaya a primera esquila (Cuadro 2). La baja heredabilidad obtenida no permite utilizar esta característica dentro los criterios de selección.

\section{Factor de Confort}

La heredabilidad del factor de confort obtenida (0.34) es moderada-alta, y coincide con los valores encontrados por otros autores (ver el Cuadro 2).

\section{Correlaciones Genéticas}

La correlación genética entre el diámetro de fibra y la desviación estándar resultó (0.80) positiva y alta (Cuadro 1 ), coincidiendo con otros autores [Cervantes et al. (2010) con 0.71; Gutiérrez et al. (2009) y Gutiérrez (2011) con 0.72 y 0.67 , respectivamente; Cruz (2011) con 0.96; Gutiérrez (2013) con 0.61; More et al. (2017) con 0.71]. Es decir, a medida que se intente disminuir el diámetro de fibra, la desviación estándar también disminuirá por tener una correlación positiva muy alta, lo que supone un cambio en el sentido favorable de ambos caracteres. También indica que si se selecciona una fibra más fina se seleccionaría indirectamente por fibras homogéneas.

La correlación genética entre el diámetro de fibra y el coeficiente de variación resultó negativa y baja (-0.06) (Cuadro 1), difiriendo del valor negativo y alto (-0.83) reportado por Cruz (2011) y del valor positivo y medio (0.32) encontrado por Renieri et al. (2009). Por otro lado, los resultados fueron similares a los reportados por otros autores [Gutiérrez (2013): -0.06; Gutiérrez et al. (2009): 0.03; Cervantes et al. (2010): 0.14]. La gran variabilidad de las correlaciones 
Cuadro 1. Heredabilidades y correlaciones genéticas para cuatro características de la fibra de alpaca utilizando el promedio de tres zonas corporales (muslo, costillar medio y paleta)

\begin{tabular}{lcc}
\hline & $\mathrm{h}^{2} \pm$ e.e. & $\mathrm{r}_{\mathrm{g}} \pm$ e.e \\
\hline Diámetro de fibra (DF) & $0.43 \pm 0.09$ & \\
Desviación estándar (DE) & $0.31 \pm 0.09$ & \\
Coeficiente de variación (CV) & $0.17 \pm 0.08$ & \\
Factor de confort (FC) & $0.34 \pm 0.09$ & \\
DF - DE & & $0.80 \pm 0.09$ \\
DF - CV & $-0.06 \pm 0.24$ \\
DF - FC & $-0.93 \pm 0.04$ \\
DE - CV & $0.68 \pm 0.14$ \\
DE - FC & $-0.94 \pm 0.03$ \\
CV - FC & $-0.36 \pm 0.21$ \\
\hline
\end{tabular}

$h^{2}$ : heredabilidad; $r_{g}$ : correlación genética; e.e: error estándar

Cuadro 2. Heredabilidades para características de la fibra de alpaca reportados en la literatura científica en alpacas Huacaya a primera esquila

\begin{tabular}{lcccc}
\hline Referencia & Diámetro & $\begin{array}{c}\text { Desviación } \\
\text { estándar }\end{array}$ & $\begin{array}{c}\text { Coeficiente } \\
\text { de variación }\end{array}$ & $\begin{array}{c}\text { Factor de } \\
\text { confort }\end{array}$ \\
\hline Ponzoni et al. $(1999)$ & $0.67(0.30)$ & $0.66(0.32)$ & $0.90(0.30)$ & \\
Renieri et al. (2009) & 0.32 & & 0.46 & \\
Gutiérrez et al. $(2009)$ & 0.41 & 0.46 & 0.37 & \\
Gutiérrez (2013) & 0.34 & 0.39 & 0.23 & 0.25 \\
More et al. (2017) & 0.48 & 0.27 & & \\
Gutiérrez (2011) & 0.24 & 0.21 & 0.09 & 0.14 \\
Cruz (2011) & 0.53 & 0.03 & 0.10 & \\
Paredes et al. (2011) & $0.36(0.20)$ & & & \\
Cervantes et al. (2010) & 0.26 & 0.42 & 0.38 & 0.26 \\
Pérez-Cabal et al. $(2010)$ & $0.36(0.15)$ & 0.38 & 0.25 & 0.23 \\
Wuliji et al. (2000) & $0.73(0.19)$ & & & \\
León-Velarde y Guerrero (2001) & $0.18(0.32)$ & & & \\
Raunelli y Coronado (2006) & 0.53 & & & \\
\hline
\end{tabular}


Cuadro 3. Estimaciones de heredabilidad y correlaciones genéticas del diámetro, desviación estándar y coeficiente de variación, y del factor de confort de la fibra de alpaca, según la zona corporal de toma de muestra

\begin{tabular}{|c|c|c|c|c|c|c|c|c|}
\hline \multirow[t]{2}{*}{ Zona corporal } & \multicolumn{2}{|c|}{ Diámetro } & \multicolumn{2}{|c|}{ Desviación estándar } & \multicolumn{2}{|c|}{$\begin{array}{c}\text { Coeficiente de } \\
\text { variación }\end{array}$} & \multicolumn{2}{|c|}{ Factor de confort } \\
\hline & $\mathrm{h}^{2} \pm \mathrm{e} . \mathrm{e}$ & $r_{g} \pm$ e.e & $\mathrm{h}^{2} \pm$ e.e & $\mathrm{r}_{\mathrm{g}} \pm$ e.e & $\mathrm{h}^{2} \pm$ e.e & $r_{g} \pm$ e.e & $\mathrm{h}^{2} \pm$ e.e & $r_{g} \pm$ e.e \\
\hline Muslo & $0.34 \pm 0.08$ & & $0.17 \pm 0.07$ & & $0.12 \pm 0.06$ & & $0.24 \pm 0.07$ & \\
\hline Costillar & $0.38 \pm 0.09$ & & $0.31 \pm 0.08$ & & $0.22 \pm 0.07$ & & $0.26 \pm 0.08$ & \\
\hline Paleta & $0.35 \pm 0.08$ & & $0.28 \pm 0.08$ & & $0.18 \pm 0.08$ & & $0.29 \pm 0.08$ & \\
\hline Muslo-costillar & & $0.97 \pm 0.02$ & & $0.97 \pm 0.07$ & & $0.79 \pm 0.11$ & & $0.85 \pm 0.05$ \\
\hline Muslo-paleta & & $0.94 \pm 0.02$ & & $0.85 \pm 0.07$ & & $0.93 \pm 0.11$ & & $0.80 \pm 0.05$ \\
\hline Costillar-paleta & & $0.91 \pm 0.02$ & & $0.72 \pm 0.07$ & & $0.54 \pm 0.11$ & & $0.69 \pm 0.05$ \\
\hline
\end{tabular}

$h^{2}$ : heredabilidad; $r_{g}$ : correlación genética; e.e: error estándar

genéticas entre diámetro de fibra y coeficiente de variación indica que se pueden trabajar como caracteres separados, ya que la selección para uno de ellos no influiría en la selección para el otro carácter.

La correlación genética entre el diámetro de fibra y el factor de confort resultó negativa y muy alta (-0.93) (Cuadro 1), similar a otros resultados [Cervantes et al. (2010): -0.97; Gutiérrez et al. (2009) y Gutiérrez (2013): -0.97, -0.78], lo cual implica que si en programa de selección se intenta disminuir el diámetro de fibra, el factor de confort aumentaría, lo que supone un cambio en el sentido favorable del carácter.

La correlación genética entre la desviación estándar y el coeficiente de variación resultó positiva y alta (0.68) (Cuadro 1), similar al 0.74 reportado por Cervantes et al. (2010) y 0.75 y 0.61 reportados por Gutiérrez et al. (2009) y Gutiérrez (2013), respectivamente, aunque difiere de la correlación negativa (-0.24) reportada por Cruz (2011). En este caso, se puede concluir que a medida que se intente disminuir la desviación estándar durante el proceso de selección genética, el coeficiente de variación también disminuiría.
La correlación genética entre la desviación estándar y el factor de confort resultó negativa y muy alta (-0.94) (Cuadro 1), siendo semejante a estudios previos [Cervantes et al. (2010): -0.83; Gutiérrez et al. (2009) y Gutiérrez (2013): -0.79 y -0.64, respectivamente]. en forma similar, la correlación genética entre el coeficiente de variación y el factor confort resultó negativa y moderada $(-0.38)$, tal y como fue demostrado previamente [Cervantes et al. (2010): -0.24; Gutiérrez (2013): -0.14]; lo cual implica, al igual que en la correlación genética entre el diámetro de fibra y el factor de confort, que conforme se logre disminuir la desviación estándar o el coeficiente de variación del diámetro de la fibra se aumentaría el factor confort.

\section{Heredabilidades y Correlaciones Gené- ticas según la Zona Corporal}

Las características independientes por cada zona corporal se presentan en el Cuadro 3. Las heredabilidades para el diámetro, desviación estándar, y factor de confort fueron moderadas en las tres zonas corporales: muslo, costillar medio y paleta. Las correlaciones genéticas fueron positivas y modera- 
das-altas con excepción de la que existe entre costillar medio y paleta para el coeficiente de variación $(\mathrm{r}=0.54)$. Estos resultados justifican el uso de la muestra del costillar medio con fines de selección por estas características, exceptuando para el coeficiente de variación.

La comparación de las heredabilidades y correlaciones genéticas de cada característica con el promedio de las tres zonas corporales y de todas las características independientes por cada zona corporal indican, en forma general, que se obtienen valor de heredabilidad más altos para diámetro de fibra, desviación estándar y factor de confort con el promedio de las tres zonas corporales, en tanto que para el coeficiente de variación es mejor trabajar con cada zona corporal.

\section{Conclusiones}

- La variabilidad fenotípica de diámetro de fibra, desviación estándar y factor de confort está influenciada por el componente genético aditivo.

- La heredabilidad del diámetro de fibra, desviación estándar y coeficiente de variación en el costillar medio fue mayor con respecto al muslo y la paleta.

- La heredabilidad de cada característica promediada en las tres zonas fue mayor que por zona de muestreo.

- La correlación genética entre el diámetro de fibra con la desviación estándar favorece la selección simultánea para finura y uniformidad de los vellones de alpaca.

\section{Agradecimientos}

Los autores expresan su agradecimiento a la Universidad Nacional Agraria la Molina (UNALM), al laboratorio de fibras «Alberto Pumayalla Díaz», al Proyecto VLIRUNALM, a FONDECYT-CONCYTEC, a MICHELL \& CIA y a la University of Natural Resources and Life Sciences (Boku,
Viena, Austria) por las facilidades brindadas para la realización del estudio.

\section{Literatura Citada}

1. Cervantes I, Pérez-Cabal M, Morante $R$, Burgos A, Salgado C, Nieto B, Goyache $F$, et al. 2010. Genetic parameters and relationships between fibre and type traits in two breeds of Peruvian alpacas. Small Ruminant Res 88: 6-11. doi: 10.1016/j.smallrumres.2009.10.016

2. Cruz L. 2011. Estimación de parámetros genéticos para caracteres productivos en alpacas (Vicugna pacos), Perú 2011. Tesis (Interuniversitaria) de Maestría. Lima, Perú: Univ. Autónoma de Barcelona. $53 \mathrm{p}$.

3. [FAO] Organización de las Naciones Unidas para la Agricultura y la Alimentación. 2005. Situación actual de los camélidos sudamericanos en Perú. Proyecto de Cooperación Técnica en apoyo a la crianza y aprovechamiento de los Camélidos Sudamericanos en la Región Andina TCP/RLA/2914. [Internet]. Disponible en: https://tarwi.lamolina.edu.pe/ emellisho/zootecnia_archivos/situacion\%-20alpcas\%20peru.pdf

4. Gilmour A, Thompson R, Cullis B. 1995. Average information REML: an efficient algorithm for variance parameter estimation in linear mixed models. Biometrics 51: 1440-1450.

5. Gutierrez JP. 2013. Fibre genetics on alpaca. $64^{\text {th }}$ Annual Meeting of the European Federation of Animal Science. Symposium on South American Camelids and other Fibre Animals. Nantes, France.

6. Gutiérrez G 2011. Valores estimados de los parámetros genéticos en poblaciones de alpacas. En: Producción y tecnología en camélidos sudamericanos. Perú: Univ. Nacional de Huancavelica. p 241-250. 
7. Gutiérrez JP, Goyache F, Burgos A, Cervantes I. 2009. Genetic analysis of six production traits in Peruvian alpacas. Livest Sci 123: 193-197. doi: 10.1016/ j.livsci.2008.11.006

8. [IWTO-12] International Wool Textile Organization. 2012. Measurement of the mean and distribution of fiber diameter using the Sirolan-laser Scan fiber diameter analyzer. Brussels: IWTO. [Internet]. Available in: http:// www.nzwta.co.nz/assets/Docs/ technical-details/fibre-diameter.pdf

9. León-Velarde C, Guerrero J. 2001. Improving quantity and quality of alpaca fibre; using a simulation model for breeding strategies. In: Third Interna-tional Symposium on Systems Approa-ches for Agricultural Development. Lima, Perú.

10. Manso C. 2011. Determinación de la calidad de fibra de alpaca en Huancavelica (Perú): Validación de los métodos de muestreo. Tesis de Ingeniero Agrónomo. España: Univ. Pública de Navarra. 121 p.

11. More M, Ponce D, Vivanco W, Asparrin M, Gutiérrez G. 2017. Genetic parameters for fleece weight and fibre characteristics in huacaya alpacas. In: World Congress on Genetics Applied to Livestock Production Digital Archive. New Zealand: Massey University.

12. Paredes M, Alonso A, Analla M, Machaca J, Mũ̃oz $A$. 2011. Genetic parameters and fixed effects estimation for fibre traits in alpaca Huacaya (Lama pacos). J Anim Vet Adv 10: 1484-1487. doi: 10.3923/javaa.2011.1484.1487

13. Pérez-Cabal MA, Cervantes I, Morante R, Burgos A, Goyache F, Gutierrez JP. 2010. Analysis of the existence of major genes affecting alpaca fiber traits. J Anim Sci 88: 3783-3788. doi: $10.2527 /$ jas.2010-2865

14. Ponzoni R, Grimson R, Hill J, Hubbard D, McGregor B, Howse A, Carmichael I, Judson G. 1999. The inheritance of and association among some production traits in young Australian alpacas. Proc Assoc Advmt Anim Breed Genet 13: 468-471.

15. Quispe E, Poma A, Purroy A. 2013. Características productivas y textiles de la fibra de alpacas de raza Huacaya. Rev Complutense Cienc Vet 7: 1-29. doi: 10.5209/rev_RCCV.2013.v7.n1.41413

16. Raunelli S, Coronado l. 2006. Un método de selección aplicable a alpacas. En: II Simposio Internacional de Investigaciones sobre Camélidos Sudamericanos. Arequipa, Perú.

17. Renieri C, Valbonesi A, La Manna V, Antonini A, Asparrin M. 2009. Inheritance of Suri and Huacaya type of fleece in Alpaca. Ital J Anim Sci 8: 8391. doi: 10.4081/ijas.2009.83

18. SAS. 2013. User's guide (Release 9.4). Cary, North Carolina, USA: SAS Institute.

19. Thompson R. 2008. Estimation of quantitative genetic parameters. P R Soc B 275: 679-686. doi: 10.1098/rspb.2007.1417

20. Wuliji T, Davis GH, Dodds KG, Turner PR, Andrews RN, Bruce GD. 2000. Production performance, repeatability and heritability estimates for live weight, fleece weight and fiber characteristics of alpaca in New Zealand. Small Ruminant Res 37: 189-201. doi: 10.1016/ S0921-4488(00)00127-9 